\title{
Preparation and quality evaluation of a volatile oil microemulsion from Flos magnoliae and Centipeda minima
}

\author{
YULIN LIANG $^{1 *}$, JUNBO ZOU $^{2 *}$, XIAOFEI ZHANG ${ }^{2}$, YAJUN SHI $^{2}$, JIA TAI $^{1}$, \\ YU WANG ${ }^{1}$, DONGYAN GUO ${ }^{2}$ and MING YANG ${ }^{3}$ \\ ${ }^{1}$ Department of Pharmaceutics and ${ }^{2}$ Shaanxi Province Key Laboratory of New Drugs and \\ Chinese Medicine Foundation Research, College of Pharmacy, Shaanxi University of Chinese Medicine, \\ Xianyang, Shaanxi 712046; ${ }^{3}$ Key Laboratory of Modern Preparation of Traditional Chinese Medicine, Ministry of Education, \\ Jiangxi University of Traditional Chinese Medicine, Nanchang, Jiangxi 330004, P.R. China
}

Received November 24, 2019; Accepted July 10, 2020

DOI: $10.3892 / \mathrm{mmr} .2020 .11571$

\begin{abstract}
In order to improve the water solubility of the volatile oils extracted from Flos magnoliae (FM) and Centipeda minima $(\mathrm{CM})$, they were prepared as a microemulsion $(\mathrm{ME})$, which were then used in the development of an FM and CM volatile oil ME for the treatment of allergic rhinitis (AR). ME was prepared by phase inversion emulsification, and the prescription factors such as emulsifier, co-emulsifier, oil phase, $\mathrm{Km}$, which represents the ratio of the mass of emulsifier to that of the co-emulsifier, and preparation factors such as temperature affecting the formation of the ME were selected according to the formation area of ME in a pseudo-ternary phase diagram. The quality of the ME was evaluated based on its appearance, particle size, Zeta potential and stability. The content of eucalyptol in ME was determined by gas chromatography-mass spectrometry (GC-MS). The cumulative permeability of the ME within $24 \mathrm{~h}$ was measured with a transdermal diffusion tester. The results revealed that the best formula for preparation of the ME was as follows: Castor oil polyoxyethylene ether (EL-40) was the emulsifier; the co-emulsifier was anhydrous ethanol; the $\mathrm{Km}$ was $2: 1$; the mixed phase of volatile oil and isopropyl myristate with mass ratio of 1:1 was used as oil phase; and the preparation temperature was $25^{\circ} \mathrm{C}$. The content of eucalyptol in the ME was $2.57 \mathrm{mg} / \mathrm{g}$, and the cumulative permeability of the ME in $24 \mathrm{~h}$ was significantly increased compared with that of the reference oil solution. The appearance of the ME was uniform,
\end{abstract}

Correspondence to: Professor Xiaofei Zhang or Professor Yajun Shi, Shaanxi Province Key Laboratory of New Drugs and Chinese Medicine Foundation Research, College of Pharmacy, Shaanxi University of Chinese Medicine, 1 Century Avenue, Xianyang, Shaanxi 712046 , P.R. China

E-mail: 2051028@sntcm.edu.cn

E-mail: 2051004@sntcm.edu.cn

*Contributed equally

Key words: Flos magnoliae, Centipeda minima, volatile oil, eucalyptol, microemulsion, in vitro infiltration and the solution was transparent. In conclusion, compared with traditional preparations, FM and CM volatile oil ME is a novel, improved and more effective preparation for the treatment of AR.

\section{Introduction}

Allergic rhinitis (AR) refers to the non-infective inflammatory disease of the nasal mucosa caused by the release of IgE-mediated histamine following exposure to allergens by atopic individuals, and involves a variety of immune active cells and cytokines (1). The clinical manifestations of AR are usually nasal congestion, rhinocnesmus, rhinorrhea and sneezing. AR is the most common chronic refractory disease reported in recent years in otorhinolaryngology, and it is also one of the prioritized diseases for prevention and treatment (2). It is mainly caused by environmental changes, seasonal alternation, temperature change, wind, precipitation, pollen and dust (3). The disease is not lethal, but the nasal symptoms cause obvious discomfort, seriously affecting the physical health of the patient, their ability to work and study, and their quality of life. AR is difficult to control and leads to numerous long-term complications that may cause more severe diseases, such as nasopharyngeal carcinoma and tympanitis (4). Therefore, the identification of a safe drug for the treatment of AR is currently a research priority (2).

Bitongning drops are recognized in Volume 6 of the Drug Standard of the Ministry of Health of the People's Republic of China (5). The drops are made by steam distillation of Flos magnoliae (FM) and Centipeda minima (CM) to extract their volatile oil or aqua aromatica (6). Bitongning drops can clear nasal orifices, and therefore they are used for treating nasal congestion, acute and chronic sinusitis, AR and colds caused by wind chill (7-9). Pharmacological experiments have indicated that the volatile oils of drugs used in Traditional Chinese Medicine (TCM) have anti-inflammatory and anti-allergenic effects, and they are currently used to treat acute and chronic rhinitis, as well as AR. In particular, the volatile oils of FM and CM serve important roles in the treatment of AR $(10,11)$. Due to the low and unstable extraction rate of volatile oil, constituents such as eucalyptol and geraniene D were easy to 
volatilize (12). The majority of TCM volatile oils are used as a raw material only, and therefore few previous studies have reported the clinical application of its single preparation. The association between compatibility and pharmacodynamics of FM and CM has been rarely studied. Our previous study revealed that the combination of volatile oil from FM and $\mathrm{CM}$ may effectively relieve local inflammatory cell infiltration and cell necrosis of nasal mucosa in the nasal cavity of rats, thereby decreasing the histamine content in blood and improving the symptoms of AR (11). In Bitongning drops, the active component is aromatic water. Although aromatic water is mostly composed of volatile oil, it is a type of solution that is nearly saturated or contains water. Compared with pure volatile oil, the volatile oil content of aqua aromatica is lower and extremely perishable, which is an impediment for its production and storage on a large scale.

As a new drug carrier, microemulsions (ME) are transparent or translucent, have low viscosity, and are isotropic and thermodynamically stable systems. ME is spontaneously formed by an oil phase, water phase, emulsifier and co-emulsifier in an appropriate ratio $(13,14)$. The particle size of $\mathrm{ME}$ ranges from 10-100 nm, and its characteristics include low viscosity, rapid absorption and targeted drug release, which can increase the solubility of volatile oil drugs, improve the bioavailability of drugs and decrease side effects (15-17). As the volatile oils of FM and CM are fat-soluble components, and their absorption is poor and their bioavailability is low, the common method of preparation results in poor absorption and low bioavailability. Therefore, in the present study, the volatile oils of FM and CM were prepared as an ME by phase conversion emulsification, and its basic properties, including stability, content and skin permeability were evaluated. The results led to the generation of a novel type of FM and CM volatile oil ME preparation with high stability, safety and loading capacity.

\section{Materials and methods}

Materials. FM volatile oil and CM volatile oil were produced by the Key Laboratory of New Drugs and Chinese Medicine Foundation Research, College of Pharmacy, Shaaxi University of Chinese Medicine (7,20). Eucalyptol (purity >99.5\%) was purchased from Shanghai Aladdin Bio-Chem Technology Co., Ltd. Hydrogenated castor oil polyoxyethylene ether (RH-40), castor oil polyoxyethylene ether (EL-40), isopropyl myristate (IPM) and isopropyl hexadecanoate (IPP) were purchased from Shanghai Yuanye Bio-Technology Co., Ltd. Tween-80, Tween-20, glycerol, 1,2-propanediol, and oleic acid were purchased from Tianjin Tianli Chemical Reagent Co., Ltd. Polyethylene glycol-400 (PEG-400) was purchased Tianjin Kemiou Chemical Reagent Co., Ltd. Sudan III and methylene blue were obtained from Shanghai Xinsheng Test Chemical Technology Co., Ltd. Purified water, purchased from Wahaha Group Co., Ltd., was used throughout the experiments. All other reagents were of commercial analytical grade. A total of three three-month-old female bullfrogs $(150 \pm 20 \mathrm{~g})$ were supplied by Chengdu Dossy Experimental Animals Co., Ltd. Bullfrogs were housed in a transparent box with a water depth of $20 \mathrm{~cm}$ at $25^{\circ} \mathrm{C}, 30-40 \%$ humidity and a 12-h light day cycle, without food for 3 days. Bullfrogs were sacrificed by marrow destruction, and the abdominal skin and subcutaneous fat was removed. The present study was approved by the Ethical Committee of Shaanxi University of Chinese Medicine.

\section{Screening of formula and preparation factors of $M E$}

Emulsifiers. Representing an important component in the preparation of MEs, an emulsifier is a substance that can improve the surface tension between various phases in emulsion, and can form a uniform and stable dispersion system or emulsion (21). The hydrophilic and lipophilic balance (HLB) value of the emulsifier is the basic metric used for selecting a certain emulsifier (22). In the present study, RH-40, EL-40, Tween-80, and Tween-20, emulsifiers with HLB values between 8-18 that can effectively improve the surface tension of oil and water, were selected to investigate their effects on the formation of volatile oil ME. The mixed phase with 1:1 ratio of IPM to FM-CM volatile oil was used as the oil phase, and the preparation temperature was $25^{\circ} \mathrm{C}$. The different ratios of emulsifier to mixed oil phase examined were 9:1, 8:2, 7:3, $6: 4,5: 5,4: 6,3: 7,2: 8$ and 1:9. ME was produced by stirring at constant temperature and uniform speed, adding ultra-pure water drop by drop and measuring the electrical conductivity. When the electrical conductivity of the system reached the highest point, it was defined as being an ME, and the dosage of emulsifier, mixed oil phase and water phase which can form the ME group was recorded (14,23). Data input was analyzed using Origin 8.0 drawing software (OriginLab Corporation) to generate pseudo-ternary phase diagrams.

Co-emulsifier. According to the screening results, EL-40 was selected as the emulsifier, the mixed phase of IPM and FM-CM volatile oil at a ratio of 1:1 was selected as the oil phase, the $\mathrm{Km}$ was $2: 1$, and the preparation temperature was $25^{\circ} \mathrm{C}$. The effects of the co-emulsifiers such as absolute ethyl alcohol, glycerol, 1,2-propanediol and PEG-400 on ME were investigated.

Oil phase. In the preparation of ME, the size and chain length of the oil phase molecules are important for the formation of ME. In the present study, IPM, IPP and oleic acid were selected as the components of the oil phase. According to the screening results of emulsifier and co-emulsifier, EL-40 was used as emulsifier, while anhydrous ethanol was used as co-emulsifier; the $\mathrm{Km}$ value was $2: 1$ and the preparation temperature was $25^{\circ} \mathrm{C}$. IPM, IPP, oleic acid and the FM-CM volatile oil of FM-CM were mixed in a certain proportion as the oil phase. The optimum oil phase for ME preparation was screened.

$K m$ value. In the preparation of $\mathrm{MR}$, the $\mathrm{Km}$ value is the ratio of emulsifier quality to co-emulsifier mass. The ability of $\mathrm{ME}$ formation varies with $\mathrm{Km}$ value. According to the screening results of the emulsifier, co-emulsifier and oil phase, EL-40 was used as emulsifier, anhydrous ethanol as co-emulsifier, and IPM and FM-CM volatile oil as mixed oil phase. The preparation temperature was $25^{\circ} \mathrm{C}$, the effects of $\mathrm{Km}$ values of 1:1, 2:1, 3:1 and 4:1 on ME formation were investigated.

Preparation temperature. Throughout the screening procedures of the above prescription factors, the basic prescription was determined, and the effects of different temperatures (25, 30,40 and $50^{\circ} \mathrm{C}$ ) on the preparation of $\mathrm{ME}$ were investigated.

Characterization of $M E$. ME was characterized as described in previous studies (15,24-27).

Appearance. The copper mesh of the carbon-plated support film was placed on the sealing film, and a drop of sample 
$(\sim 30 \mu \mathrm{l})$ was added to the support film. Following incubation for 5-10 min, the excess solution was removed from the edge of the filter paper with a pointed sheet of filter paper, and placed on the filter paper for $1 \mathrm{~min}$ and allowed to drain. Then, the dried supporting film was placed on the sealing film, and a drop of uranyl acetate dye solution was added for $90 \mathrm{sec}$. Subsequently, the excess dye solution was removed with a pointed filter paper, clip it on the filter paper and dried for $3 \mathrm{~h}$. A JEM-1200EX transmission electron microscope (JEOL, Ltd.) was used for observation, as described previously (26).

Type identification. According to the principle of 'similar miscibility', $1 \mathrm{~g} / \mathrm{l}$ of sudan III (an oil-soluble dye) and methylene blue (a water-soluble dye) solution were added at room temperature, and their diffusion rates in ME were directly observed by the naked eye for $1 \mathrm{~min}$ to determine ME. When the ME was of the water in oil type, the diffusion rate of Sudan III was increased compared with that of methylene blue, and when the $\mathrm{ME}$ was of the oil in water type, the diffusion rate of methylene blue was increased compared with that of Sudan III.

$p H$ and refractive index. Substances delivered by nasal administration must have a suitable $\mathrm{pH}$ value, and the $\mathrm{pH}$ value of the ME in turn affects the stability of the system. The $\mathrm{pH}$ and refractive index of the freshly prepared $\mathrm{ME}$ were measured using a PHS-3C pH meter (Shanghai Yidian Scientific Instruments Co., Ltd.) and WYA-2W Abbe refractometer (Shanghai Instrument Electrophysical Optical Instruments Co., Ltd.). At room temperature $\left(25^{\circ} \mathrm{C}\right)$, ultra-pure water was used as the control for calibration. In total, the $\mathrm{pH}$ values of 3 batches of ME were determined, and the average value was calculated.

Particle size and Zeta potential. In total, 3 batches of $1 \mathrm{ml}$ MEs were selected, and the particle size and zeta potential of the freshly prepared MEs were determined by Malvern Zetasizer NanoZS90 instrument (Malvern Instruments Ltd.). According to the basic definition of $\mathrm{ME}$, the particle size of $\mathrm{ME}$ should be between $10-100 \mathrm{~nm}$ (28). Zeta potential measurement is the use of electrophoretic scattering to detect the potential of suspended particles in a specific solution environment. Its purpose is to detect the charged properties of the particle surface, including electrical properties and potential level, in order to predict the stability of the whole suspension system.

Physical stability. In total, 3 batches of the same ME quantity were placed in a centrifuge tube in a 416 low-speed centrifuge (Gene Company, Ltd.) and centrifuged at $1,890 \mathrm{x} \mathrm{g}$ for $30 \mathrm{~min}$ at $4^{\circ} \mathrm{C}$. Following centrifugation for $30 \mathrm{~min}$, the appearance of ME was observed.

Thermodynamic stability. A total of 3 batches of ME were subjected to a heating-cooling cycle experiment. The $10 \mathrm{ml}$ $\mathrm{ME}$ was placed in a centrifuge tube. Following continuous heating-cooling cycles between 40 and $4^{\circ} \mathrm{C}$ for 6 times, its appearance was observed.

In total, 3 batches of $\mathrm{ME}$ were subjected to a freezing-thawing cycle experiment. The $10 \mathrm{ml} \mathrm{ME}$ was placed in a centrifuge tube and frozen in the refrigerator at $-20^{\circ} \mathrm{C}$ for $24 \mathrm{~h}$. Upon thawing at room temperature for $24 \mathrm{~h}$, after 6 consecutive cycles, its appearance was observed.

\section{Detection of eucalyptol in $M E$}

Preparation of reference substance. The weight of the eucalyptol reference substance was $0.2541 \mathrm{~g}$, the volume of anhydrous ether was set as $5 \mathrm{ml}$ as the reference substance solution, and the concentration of the reference liquid was $50.82 \mathrm{mg} / \mathrm{ml}$.

Preparation of samples. Weighed ME (1.0 g in $5 \mathrm{ml})$ was added to a brown flask, followed by $5 \mathrm{ml}$ of anhydrous ether. Following demulsification with Eddy for $1 \mathrm{~min}$, centrifugation at $1,890 \mathrm{x} \mathrm{g}$, at $4^{\circ} \mathrm{C}$ for $10 \mathrm{~min}$ and filtration of the supernatant through a $0.22 \mu \mathrm{m}$ microporous filter membrane, the sample solution was obtained.

Gas chromatography-mass spectrometry (GC-MS). An Agilent 7890GC/5977MS (Agilent Technologies, Inc.) was used to investigate the subsequent experimental methodology and the content of Eucalyptol, according to the manufacturer's protocol. In addition, the content of eucalyptol in ME was determined.

Chromatographic conditions. The chromatographic column used in the present study was a HP-5 quartz capillary column (30 m x $0.25 \mathrm{~mm}, 0.25 \mu \mathrm{m})$. The temperature of the injection port was $250^{\circ} \mathrm{C}$. The initial temperature of the oven was $55^{\circ} \mathrm{C}$ (held for $2 \mathrm{~min}$ ), with an increase of $8^{\circ} \mathrm{C} / \mathrm{min}$ until it reached $80^{\circ} \mathrm{C}$ (held for $0 \mathrm{~min}$ ), followed by an increase of $6^{\circ} \mathrm{C} / \mathrm{min}$ until reaching $160^{\circ} \mathrm{C}$ (held for $2 \mathrm{~min}$ ), and then an increase of $8^{\circ} \mathrm{C} / \mathrm{min}$ until reaching $200^{\circ} \mathrm{C}$ (held for $0 \mathrm{~min}$ ), with an increase of $3^{\circ} \mathrm{C} / \mathrm{min}$ until reaching a final temperature of $250^{\circ} \mathrm{C}$ (held for $3 \mathrm{~min}$ ). The total run time was $42.25 \mathrm{~min}$. The sample was injected at 40:1 with high-purity helium as carrier gas and a constant flow rate of $1 \mathrm{ml} / \mathrm{min}$.

Mass spectrometry conditions. The ion source was an EI source, the multiplication voltage was $1.5 \mathrm{kV}$, the electron energy was $70 \mathrm{EV}$, the mass scanning range was $28-555 \mathrm{~m} / \mathrm{z}$, and the solvent delay was $3 \mathrm{~min}$.

Methodological investigation. The eucalyptol reference solution was prepared by pouring $25,50,100,200,400$ or $800 \mu \mathrm{l}$ of eucalyptol solution into brown volumetric bottles. Then anhydrous ether was added to a final volume of $10 \mathrm{ml}$ to prepare reference solutions with concentrations of $0.12705,0.2541$, $0.5082,1.0164,2.0328$ and $4.0656 \mathrm{mg} / \mathrm{ml}$ were prepared. Linear regression was performed using the concentration and peak area as abscissa and longitudinal coordinates with Microsoft Excel 2016 (Microsoft Corporation).

The same sample solution was injected repeatedly 6 times according to the above conditions. The relative standard deviation (RSD) of the peak area of eucalyptol was determined, and the precision of the method was investigated. In total, 6 samples were prepared in parallel with the same batch of sample solutions. According to the aforementioned conditions, the RSD of the peak area of eucalyptol was calculated, and the experiment method was repeated six times to investigate the repeatability. The same batch of ME solution was prepared according to the treatment method of the test sample, and the peak area of eucalyptol was determined at $0,1,2,4,6$, $8,10,12$ and $24 \mathrm{~h}$. The peak area of eucalyptol RSD was calculated to investigate the stability of the ME within $24 \mathrm{~h}$. A total of 9 samples of $0.5000 \mathrm{~g} \mathrm{ME}$ were precisely weighed and divided into 3 groups. Eucalyptol reference solution was added according to the known levels of 50, 100 and $150 \%$, respectively. Following mixing, the samples were analyzed according to the above chromatographic conditions, and the recovery and RSD value were calculated. 

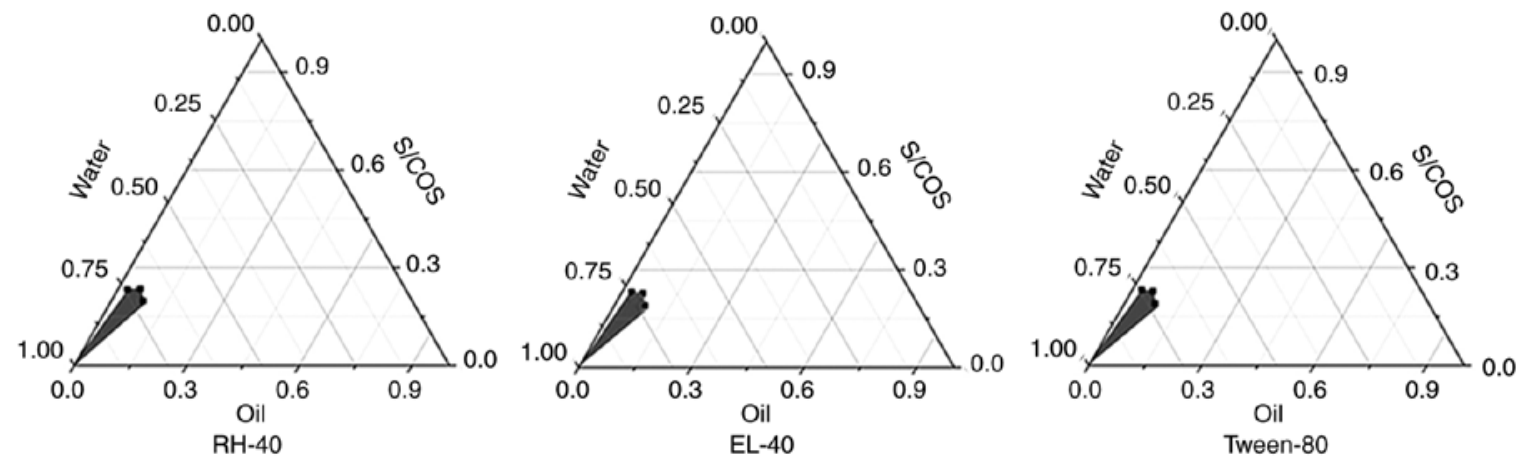

Figure 1. Pseudo-ternary phase diagram of different microemulsion emulsifiers.

Determination of eucalyptol. In total, 3 batches of samples were combined into a sample solution according to the treatment method of the sample solution, and the determination was performed according to the above chromatographic conditions.

Percutaneous permeability test. A marrow-destroying needle was inserted into the foramen magnum of the bullfrog, the spinal cord was transected left and right, and the brain was destroyed. The myelocytic needle was then withdrawn from the foramen magnum and inserted into the spinal canal to destroy the spinal cord. Death was confirmed by checking for complete relaxation of the limbs muscles. The abdominal skin of bullfrog was removed, and the fat and subcutaneous tissues were stripped, washed repeatedly with normal saline and fixed on the diffusion interface of the diffusion chamber, while the dermis was fixed to the reception tank. As the receptor fluid, 20\% ethanol saline (Kunshan Hechuang Ultrasonic Instruments Co., Ltd) was used, which was injected into the reception tank following ultrasonic cleaning to remove bubbles. The temperature of the circulating water bath was $37 \pm 0.2^{\circ} \mathrm{C}$, and a constant speed of $350 \mathrm{rpm}$ was employed to drain the bubbles in the receiving solution. Following balancing of the water bath for $30 \mathrm{~min}, 2.0 \mathrm{~g} \mathrm{ME}$ and $2.0 \mathrm{~g}$ oil solution were placed into the supply tank. At $0.5,1,2,4,6,8,10,12$ and $24 \mathrm{~h}, 1 \mathrm{ml}$ was sampled, and the same volume of blank receptor fluid was added to the reception tank and the bubbles were excreted (25,29-32). The ME solution was extracted with anhydrous ether 3 times, and the supernatant was collected and combined. The supernatant was centrifuged for $10 \mathrm{~min}$ in a high-speed centrifuge $\left(335 \mathrm{x} \mathrm{g}, 4^{\circ} \mathrm{C}\right), 1.5 \mathrm{ml}$ supernatant was extracted with a disposable syringe and passed through a filter membrane of $0.22 \mu \mathrm{m}$, and the receiving solution at each timepoint was obtained. The content of eucalyptol was determined according to the above chromatographic conditions, and the data were recorded. The cumulative transdermal volume was calculated according to the following formula:

$$
\mathrm{Qn}=\left(\mathrm{V} \times \mathrm{Cn}+\sum_{\mathrm{i}-1}^{\mathrm{n}-1} \mathrm{x} \mathrm{Vi} \times \mathrm{Ci}\right) / \mathrm{A}
$$

where $\mathrm{Qn}$ is the cumulative permeability per unit area at the time $\mathrm{n}, \mathrm{A}$ is the effective transdermal area (superficial area $=1.54 \mathrm{~cm}^{2}$ and diameter $=1.4 \mathrm{~cm}$ ), $\mathrm{Cn}$ is the concentration of the drug measured at time $\mathrm{n}, \mathrm{V}$ is the volume of the receiving pool $(15 \mathrm{ml})$, and $\mathrm{Vi}$ is the volume of each sample.
Cumulative transmittance was calculated using the formula $\mathrm{Ln}=\mathrm{Qn} / \mathrm{W}$, where $\mathrm{Ln}$ is the cumulative transmittance and $\mathrm{W}$ is the content of eucalyptol in the sample.

\section{Results}

Screening of prescription and preparation factors of $M E$ Emulsifiers. The data from the present study were collated to draw a pseudo-ternary phase diagram, as shown in Fig. 1, where the area of ME accounts for $\mathrm{S}_{\mathrm{RH}-40}=0.0283$, $\mathrm{S}_{\mathrm{EL}-40}=0.0298$ and $\mathrm{S}_{\text {Tween-80 }}=0.0287$. The Tween-20 group could not form ME, and therefore the pseudo-ternary phase diagram could not be generated. The proportion of ME area in the EL-40 group was the largest. Therefore, EL-40 was selected as the emulsifier of FM-CM volatile oil ME.

Co-emulsifiers. A co-emulsifier can adjust the HLB value of the emulsifier and form smaller droplets, and an auxiliary emulsifier can improve the formation of an ME. The results of pseudo-ternary phase diagram in Fig. 2 indicate that the area of ME was $S_{\text {anhydrous ethanol }}=0.0782, S_{1,2 \text {-propanediol }}=0.0417$, $\mathrm{S}_{\text {glycerol }}=0.0414$ and $\mathrm{S}_{\mathrm{PEG}-400}=0.0522$. Anhydrous ethanol had the largest proportion of ME area; thus, it was selected as the co-emulsifier of FM-CM volatile oil ME.

Oil phase. The results of pseudo-ternary phase diagram showed that the proportion of $\mathrm{ME}$ area was $\mathrm{S}_{\mathrm{IPP}}=0.0530$, $\mathrm{S}_{\text {IPM }}=0.0766$ and $\mathrm{S}_{\text {oleic acid }}=0.0441$. As the proportion of $\mathrm{ME}$ area was the largest in the IPM group, this was selected as the oil phase of FM-CM volatile oil ME. The results are presented in Fig. 3.

Km values. Different $\mathrm{Km}$ values have different effects on the formation of ME. According to the results of the pseudo-ternary phase diagram in Fig. 4, the area of ME was $\mathrm{S}_{1: 1}=0.0450, \mathrm{~S}_{2: 1}=0.0766, \mathrm{~S}_{3: 1}=0.0540$ and $\mathrm{S}_{4: 1}=0.0398$. When $\mathrm{Km}$ was 2:1, the ME area was the largest; thus, 2:1 was determined to be the best $\mathrm{Km}$ value.

Preparation temperature. By generating a pseudo-ternary phase diagram, the area of $\mathrm{ME}$ was $\mathrm{S}_{25^{\circ} \mathrm{C}}=0.0766, \mathrm{~S}_{30^{\circ} \mathrm{C}}=0.0696$, $\mathrm{S}_{40^{\circ} \mathrm{C}}=0.0650$ and $\mathrm{S}_{50^{\circ} \mathrm{C}}=0.0613$, suggesting that $25^{\circ} \mathrm{C}$ was the best preparation temperature. The results are shown in Fig. 5.

Preparation of optimal prescription ME. According to the above screening results, the best prescription of FM-CM volatile oil ME was as follows: Th emulsifier was EL-40; the co-emulsifier was anhydrous ethanol; the $\mathrm{Km}$ was $2: 1$; and IPM and FM-CM volatile oil were used as mixed oil phase at $1: 1$. The mixed emulsifier accounted for $25.81 \%$; the 


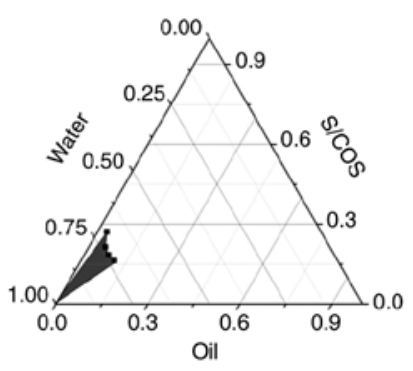

Anhydrous ethanol

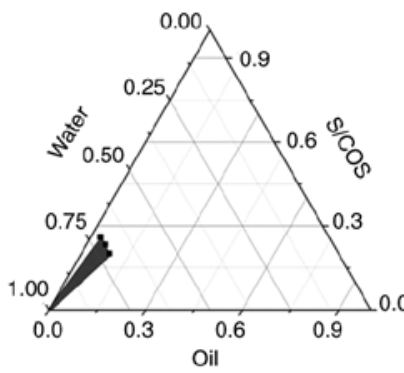

1, 2-propanediol

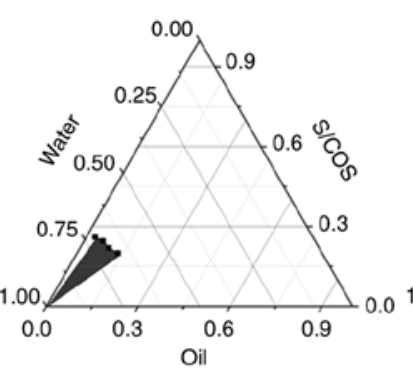

Glycerol

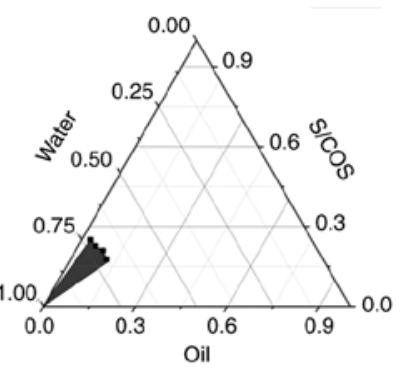

Polyethylene glycol-400

Figure 2. Pseudo-ternary phase diagram of different microemulsion co-emulsifiers.

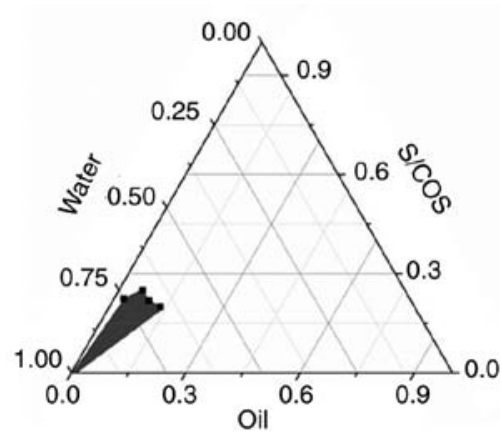

IPP

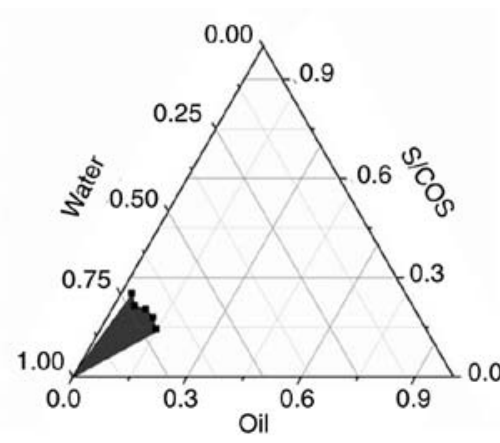

IPM

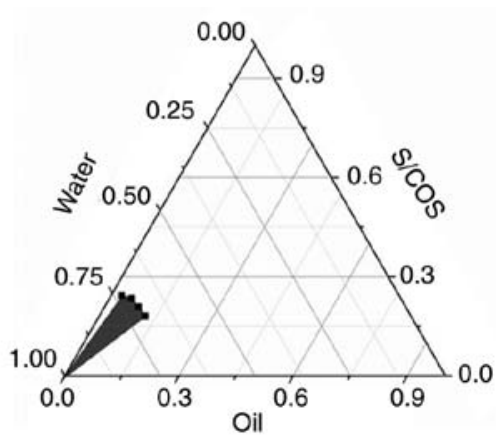

Oleic acid

Figure 3. Pseudo-ternary phase diagram of different microemulsion oil phases.
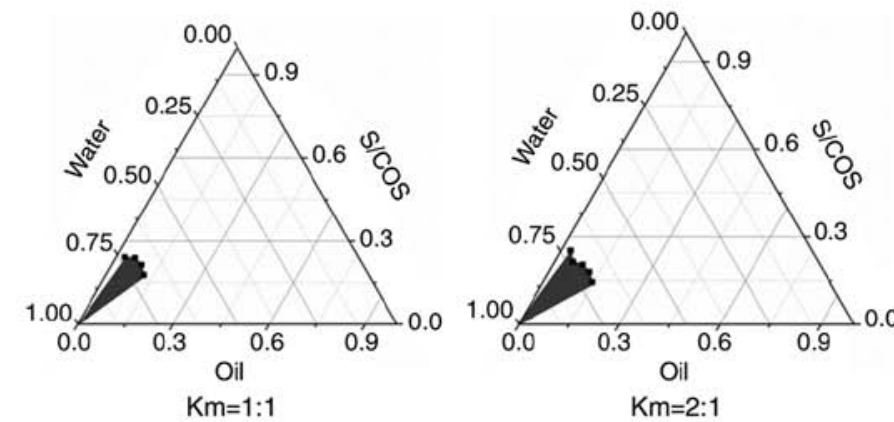

$\mathrm{Km}=2: 1$

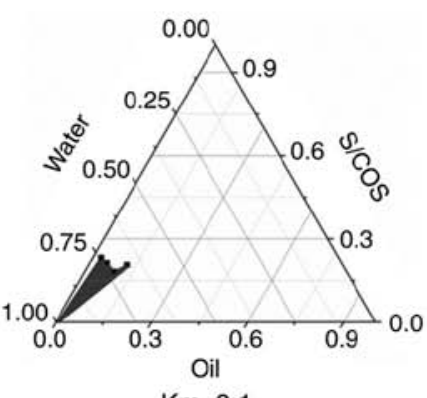

$\mathrm{Km}=3: 1$

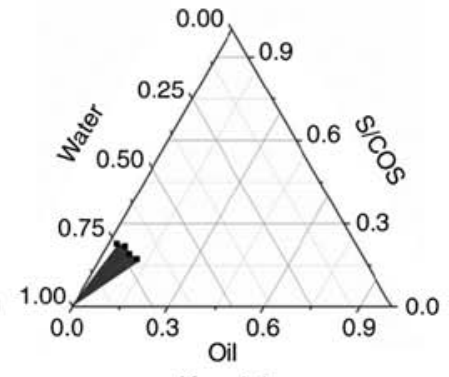

$\mathrm{Km}=4: 1$

Figure 4. Pseudo-ternary phase diagram with different microemulsion $\mathrm{Km}$ values.

mixed oil phase accounted for $2.81 \%$; and the aqueous phase accounted for $71.38 \%$ of the total ME system. The preparation temperature was $25^{\circ} \mathrm{C}$. The water representing $71.38 \%$ of the system was slowly added to form clear and transparent $\mathrm{ME}$ droplets.

\section{Characterization}

Morphological observation. The results of the observation of the appearance and morphology of the ME are presented in Fig. 6. The ME was a sphere with a round appearance and uniform particle size distribution.

Identification of ME type. The methylene blue diffusion rate of the ME prepared in the present study was markedly increased compared with that of Sudan III, which indicates that it was an O/W ME (Fig. 7).

$p H$ and refractive index. The $\mathrm{pH}$ value of $\mathrm{ME}$ was 5.67 \pm 0.01 and the refractive index was $1.4198 \pm 0.0011$ nd at $25^{\circ} \mathrm{C}$.
Particle size and Zeta potential. As shown in Fig. 8, the particle size distribution of ME was uniform and the average particle size was $14.62 \pm 0.4576 \mathrm{~nm}$. The mean polydispersity index value was $0.0747 \pm 0.0265(\mathrm{n}=3)$, and when there was only one peak in the range of $10-100 \mathrm{~nm}$, the average potential was $-4.06 \pm 0.0702 \mathrm{mV}(\mathrm{n}=3)$. These results indicated that the $\mathrm{ME}$ met the requirement of particle size, and the system was stable.

Physical stability. The appearance of the 3 batches of ME following high-speed centrifugation for $30 \mathrm{~min}$ remained uniform, clear, and transparent, and there was no stratification observed, demonstrating that the centrifugal stability of $\mathrm{ME}$ was good (Fig. 9A).

Thermodynamic stability. After 6 heating-cooling and freezing-thawing cycles, the 3 batches of ME remained clear and transparent, and there was no stratification, indicating that the thermodynamic stability of ME was good (Fig. 9B). 

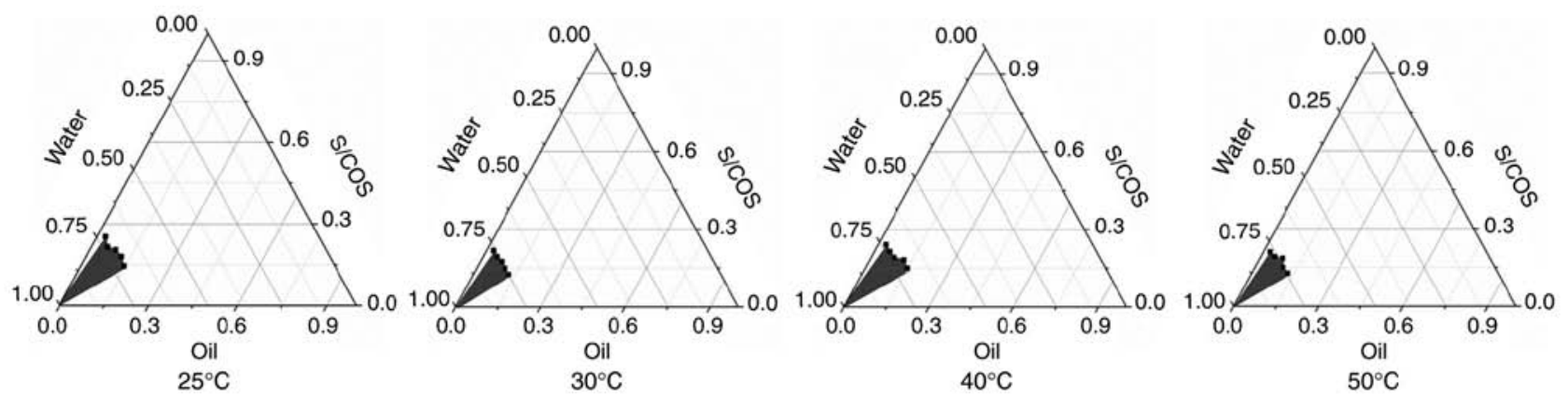

Figure 5. Pseudo-ternary phase diagram of different microemulsion preparation temperatures.

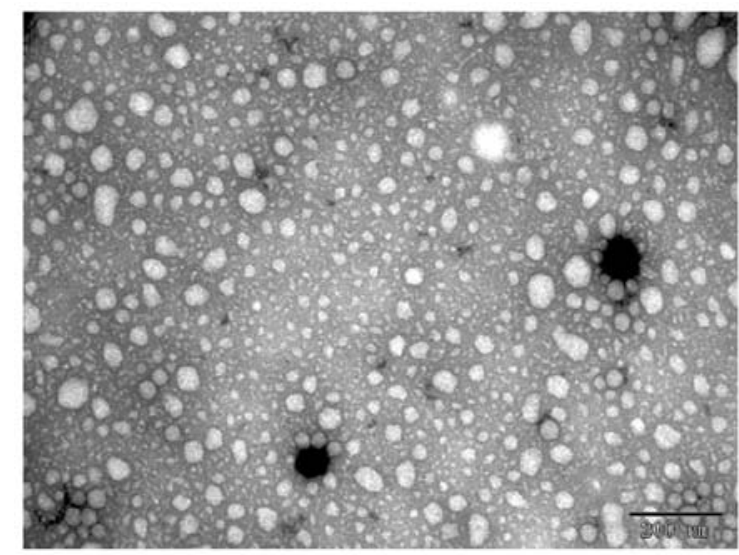

Figure 6. Transmission electron micrograph.

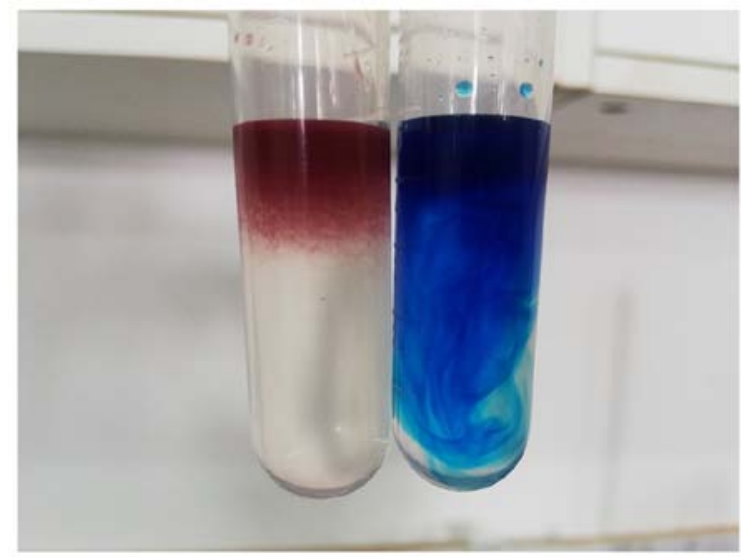

Figure 7. Identification of microemulsion type.
Methodological investigation. The calibration curve of the peak area and concentrations for eucalyptol was linear, ranging from $0.12705-4.0656 \mathrm{mg} / \mathrm{ml}$. The calibration curve was $\mathrm{Y}=37,4632 \mathrm{X}+2 \times 10^{8}\left(\mathrm{R}^{2}=0.9992\right)$, where $\mathrm{Y}$ represents the eucalyptol peak area and $\mathrm{X}$ represents the concentration of eucalyptus reference substance. The chromatograms of the reference substance and the sample solution are shown in Fig. 10. In the precision experiment, the RSD of the eucalyptol peak area was $1.36 \%$, demonstrating that the precision of this method was good. In the repeatability experiment, the RSD of the eucalyptol peak area was $2.20 \%$, indicating that this method has good repeatability. In the stability experiment, the RSD of the eucalyptol peak area was $1.93 \%$, indicating that the sample remained stable for $24 \mathrm{~h}$. In the sample recovery experiment, the mean recovery rate was $99.27 \%$, and the RSD value was $2.91 \%$. These results showed that the accuracy of the experimental observations was high.

Determination of eucalyptol concentration. According to the results of sample content determination, the mean content of eucalyptol in the ME was $2.57 \mathrm{mg} / \mathrm{g}$, as shown in Table I.

Percutaneous permeability test. The curve was generated by considering the cumulative permeability $\mathrm{Q}_{24}\left(\mu \mathrm{g} / \mathrm{cm}^{2}\right)$ as the longitudinal coordinate and the sampling time $t$ as the abscissa. Linear regression analysis of the obtained curve was performed. The linear slope represents the steady penetration rate $J_{\mathrm{Ss}}\left(\mu \mathrm{g} / \mathrm{cm}^{2} / \mathrm{h}\right)$. The results are shown in Table II, Fig. 11 and Table III.
These results indicated that the cumulative permeabilities of eucalyptol in the ME and oil solution were 301.0800 \pm 2.80 and $73.3491 \pm 2.19 \mu \mathrm{g} / \mathrm{cm}^{2}$, respectively, and the steady state penetration rates were 9.4349 and $2.0082 \mu \mathrm{g} / \mathrm{cm}^{2} / \mathrm{h}$, respectively. The cumulative permeability of the ME was increased 4.10-fold compared with that of the oil solution, and the steady penetration rate was increased 4.70 -fold compared with that of the oil solution, which indicated that ME could effectively promote the transdermal absorption of the drugs.

\section{Discussion}

Bitongning drops serve an important role in the clinical treatment of AR due to their active ingredient, the aromatic water of FM and CM, and previous studies have shown that their therapeutic effect on AR is due to their volatile oil components (33-37). The aim of the present study was to increase the drug concentration in a standard AR treatment method by replacing traditional aromatic water with volatile oil. Volatile oils have an important therapeutic effect in numerous TCM treatments, and are an indispensable functional ingredient (38). According to the theory of TCM, volatile oils function as 'aromatic strings'. An aromatic string is called 'Fangxiangzouchuan' in TCM. It can be interpreted as 'the fragrance of the medicine can be dispersed everywhere', the curative effect is exact (39). However, volatile oils are a fat-soluble component with poor water solubility that can be highly irritable. In the preliminary experiments in the present study, the best compatibility ratio of the volatile oils from FM 
Table I. Eucalyptol content in microemulsions.

\begin{tabular}{lccccccc}
\hline $\begin{array}{l}\text { Sample } \\
\text { number }\end{array}$ & $\begin{array}{c}\text { Sampling } \\
\text { quantity, }\end{array}$ & A1 & A2 & $\bar{A}$ & $\begin{array}{c}\text { Eucalyptol } \\
\text { content, mg/g }\end{array}$ & $\begin{array}{c}\text { Average } \\
\text { content, mg/g }\end{array}$ & RSD, \% \\
\hline 1 & 1.0167 & 412958743 & 383474502 & 398216622.5 & 2.60 & 2.57 & 1.19 \\
2 & 1.0012 & 381962376 & 405573769 & 393768072.5 & 2.54 & & \\
3 & 1.0032 & 398652865 & 391974721 & 395313793 & 2.56 & & \\
\hline
\end{tabular}

$\mathrm{A}_{1} ; \mathrm{A}_{2} ; \overline{\mathrm{A}} ; \mathrm{RSD}$, relative standard deviation.
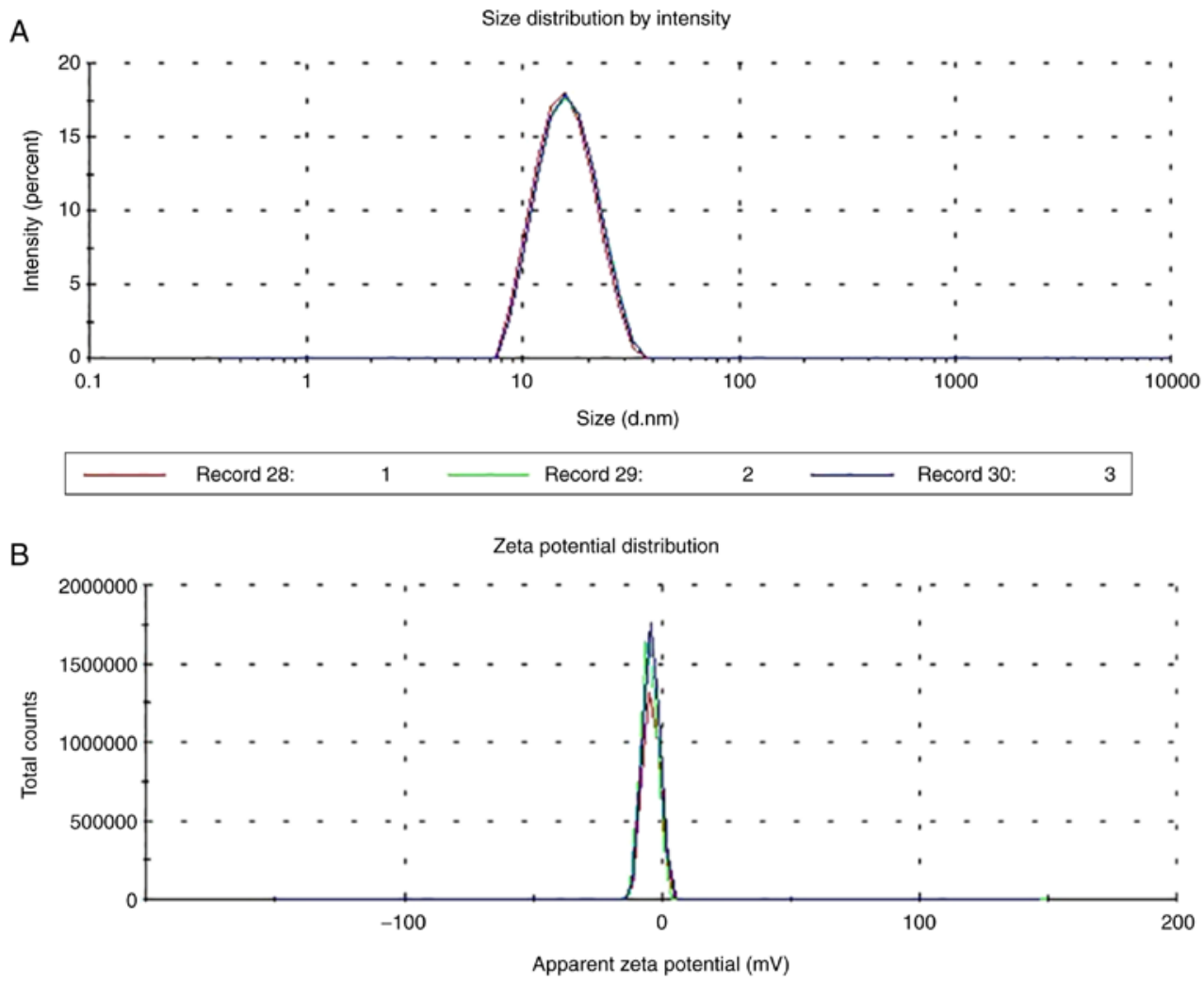

\begin{tabular}{llllllll}
\hline Record 43: $\quad 1$ & Record 44: $\quad 2 \quad$ Record 45: & 3 \\
\hline
\end{tabular}

Figure 8. Particle size and Zeta potential distribution of ME. (A) Particle size distribution of ME. (B) Zeta potential distribution of ME. ME, microemulsion.

A

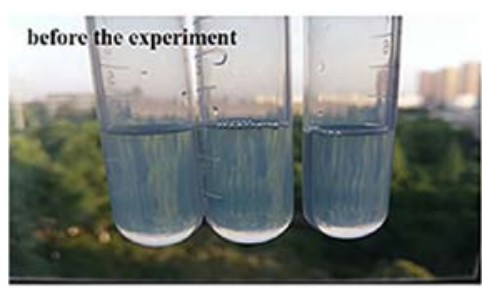

B

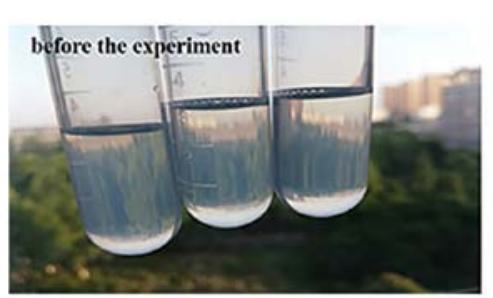

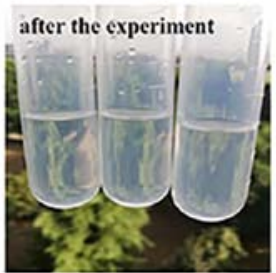

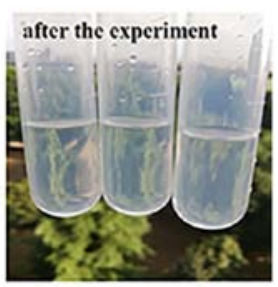

Figure 9. Thermodynamic stabilization of microemulsion.

and $\mathrm{CM}$ in the treatment of $\mathrm{AR}$ was screened, and the results revealed that the best proportion was 7:1 FM: CM. In the present study, an ME was generated to improve bioavailability, to resolve the issues of poor absorption and low solubility normally associated with volatile oils, and to decrease drug toxicity, irritation, and adverse reactions.

The ME was prepared by water titration with different emulsifiers, co-emulsifiers, oil phases, $\mathrm{Km}$ values, and preparation temperatures. The area of the ME was mapped using a pseudo-ternary phase diagram, and the particle size was combined with the size of the ME The best formula for the preparation of ME was determined by centrifugal stability and other factors. When the Km value increased from 1.0 to 2.0, the area of ME increased, which was primarily due to the increase of emulsifiers ratio, emulsifiers can effectively reduce oil/water interfacial tension and decrease the interfacial film tension formed by ME, thereby enhancing the emulsification ability to 
Table II. Qn and Ln per unit area of ME at each time-point $(\mathrm{n}=3)$.

\begin{tabular}{|c|c|c|c|c|}
\hline \multirow[b]{2}{*}{ Time-point, $\mathrm{h}$} & \multicolumn{2}{|c|}{ Eucalyptol Qn, $\mu \mathrm{g} / \mathrm{cm}^{2}$} & \multicolumn{2}{|c|}{$\mathrm{Ln}, \%$} \\
\hline & ME group & Oil solution group & ME group & Oil solution group \\
\hline 0.5 & $54.2507 \pm 0.4277$ & $21.3442 \pm 1.0544$ & 1.06 & 0.42 \\
\hline 1 & $85.4878 \pm 3.2467$ & $31.9255 \pm 3.0570$ & 1.66 & 0.62 \\
\hline 2 & $101.6378 \pm 1.1280$ & $36.3763 \pm 1.2314$ & 1.98 & 0.71 \\
\hline 4 & $120.9467 \pm 1.5481$ & $39.6531 \pm 0.4615$ & 2.35 & 0.77 \\
\hline 6 & $133.4428 \pm 1.4452$ & $42.5442 \pm 0.3600$ & 2.60 & 0.83 \\
\hline 8 & $145.0037 \pm 3.2474$ & $45.3398 \pm 0.3371$ & 2.82 & 0.88 \\
\hline 10 & $160.0303 \pm 0.4325$ & $50.6580 \pm 1.5446$ & 3.11 & 0.99 \\
\hline 12 & $173.7156 \pm 3.5513$ & $60.7943 \pm 0.3699$ & 3.38 & 1.18 \\
\hline 24 & $301.0797 \pm 2.8009$ & $73.3491 \pm 2.1919$ & 5.86 & 1.43 \\
\hline
\end{tabular}

Data are presented as the mean \pm standard deviation. Qn, cumulative permeability; Ln, cumulative transmittance; ME, microemulsion.
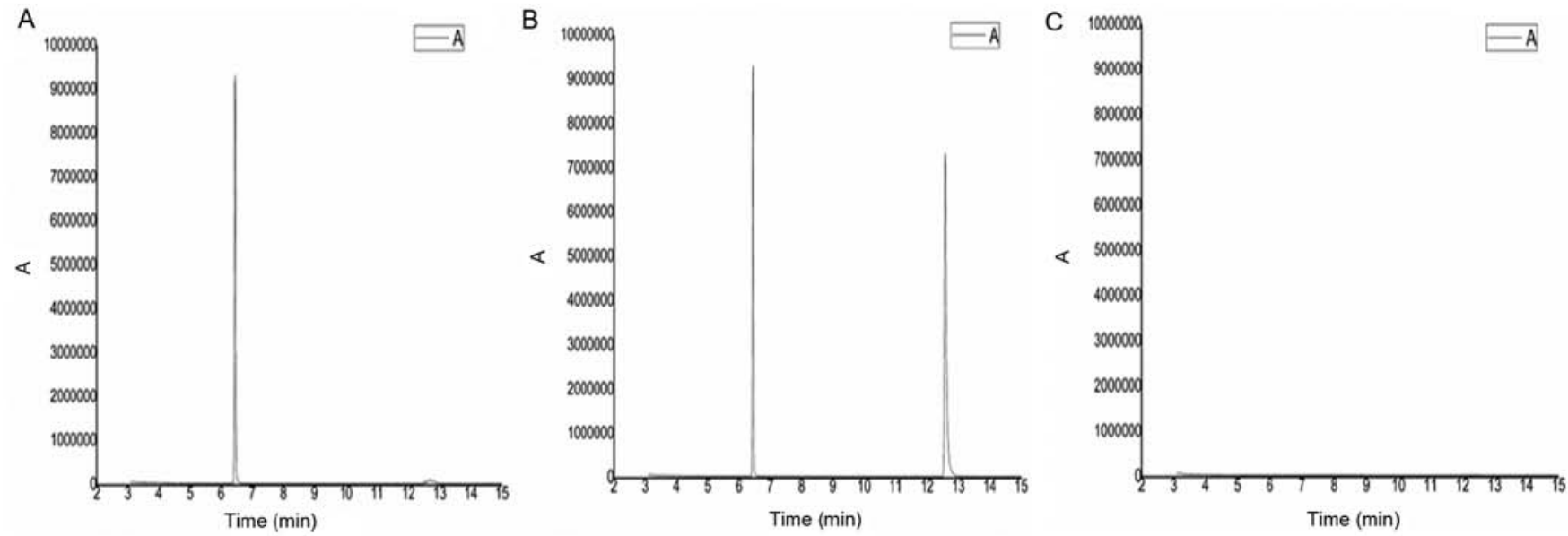

Figure 10. Gas chromatography-mass spectrometry chromatogram. (A) Reference substance. (B) Microemulsion sample. (C) Blank solvent sample. A, peak area

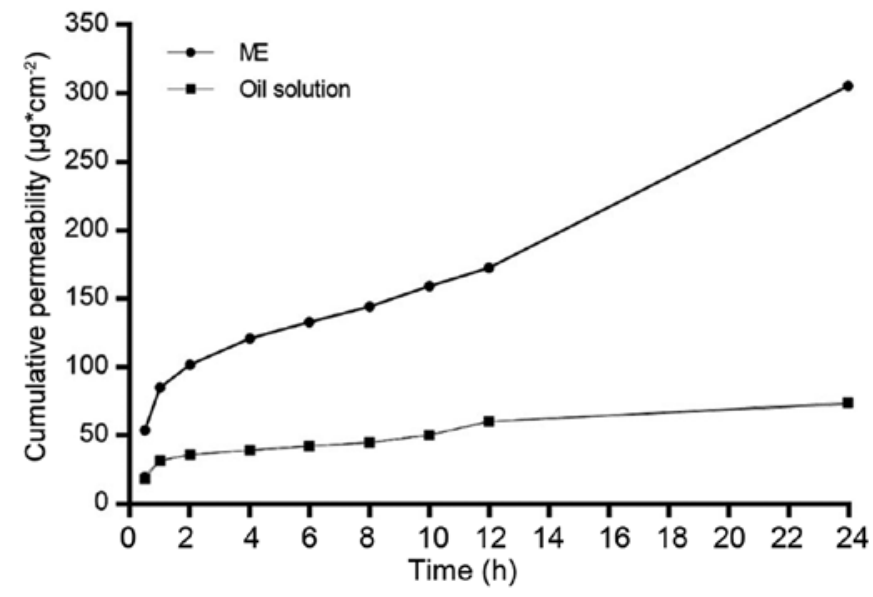

Figure 11. Percutaneous permeation curve of microemulsion and oil solution. Data are presented as the mean \pm standard deviation $(n=3)$.

form a larger ME area. When studying $\mathrm{Km}$, it has been observed that when the Km value increased from 2.0 to 4.0, possibly due to the increase of emulsifiers to a certain value, an increase in viscosity and expansion of the gel region and double continuous zone were observed, which led to the decrease of the formation area of ME (40). When examining preparation temperature, the area of ME formation was observed to decrease with the increase in preparation temperature, since volatile oil was used as part of the mixed oil phase. It was hypothesized that this may have been caused by the increase in volatilization of the drug with the increase in temperature. Therefore, $25^{\circ} \mathrm{C}$ was selected as the most suitable temperature for ME preparation. However, this hypothesis requires further experimental verification. The ME was identified as an $\mathrm{O} / \mathrm{W}$ ME, and its particle size, $\mathrm{pH}$ value and refractive index all met the requirements of an ME. It is generally considered that the absolute value of Zeta potential in a stable-dispersion system should be $>30 \mathrm{mV}$, and the larger the absolute value is, the more stable the system is (41). However, the absolute value of Zeta potential in the present study was $<30 \mathrm{mV}$. It has been reported that the Zeta potential of ME may be low (41), which does not indicate that $\mathrm{ME}$ is unstable. Instead, the primary reason for this is that the $\mathrm{ME}$ is an uncharged system. The emulsifier EL-40 in ME is a non-ionic solubilizer, which can be used in the emulsification of vegetable, animal, and mineral oils. It can also effectively 
Table III. Comparison of transdermal permeation parameters between ME and oil solution groups $(\mathrm{n}=3)$.

\begin{tabular}{lccc}
\hline Group & $\begin{array}{c}\text { Cumulative permeability - } \\
\text { time equation }\end{array}$ & $\begin{array}{c}\text { Steady penetration rate } \\
J \mathrm{ss}, \mu \mathrm{g} / \mathrm{cm}^{2} / \mathrm{h}\end{array}$ & $\begin{array}{c}\text { Cumulative permeability, } \\
\mu \mathrm{g} / \mathrm{cm}^{2}\end{array}$ \\
\hline ME group & $\mathrm{Q}=9.4349 \mathrm{t}+70.971\left(\mathrm{R}^{2}=0.9758\right)$ & 9.4349 & $301.0800 \pm 2.80$ \\
Oil solution group & $\mathrm{Q}=2.0082 \mathrm{t}+29.604\left(\mathrm{R}^{2}=0.9119\right)$ & 2.0082 & $73.3491 \pm 2.19$ \\
\hline
\end{tabular}

Data are presented as the mean \pm standard deviation. ME, microemulsion.

promote the transdermal absorption of drugs and decrease skin irritation (42). The co-emulsifier anhydrous ethanol may decrease the polarity of the aqueous phase and surface tension, thus compensating for the poor fluidity of the oil-water interface film when a emulsifier is used alone, and when combined with emulsifiers, the preparation of ME is improved $(43,44)$. Previous studies reported that adding the volatile oil of TCM to the oil phase of $\mathrm{ME}$ could improve transdermal permeation (45-47). In the present study, according to preliminary experiments, the drug with mass ratio of 1:1 and IPM were used as mixed oil phase to promote drug absorption. During the in vitro transdermal comparison between $\mathrm{ME}$ and oil solution in the present study, it was identified that the cumulative and steady permeabilities of ME were significantly increased compared with those of the oil solution group, indicating that ME may effectively promote the transdermal absorption of volatile oil. These results provide a reference for follow-up development and research of ME.

In the present study, the preparation method and quality of ME were examined systematically. It was identified that the in vitro transdermal performance of $\mathrm{ME}$ was significantly increased compared with that of the oil solutions, and that its properties were stable. In order to further demonstrate the good bioavailability of $\mathrm{ME}$, in vivo pharmacokinetic studies or cell experiments will be conducted in the future. Notably, in the preparation process of $\mathrm{ME}$, the specific formula used and preparation process factors have important effects on the formation of ME. Consequently, the selection of the appropriate emulsifier, co-emulsifier, oil phase, $\mathrm{Km}$, and preparation temperature are important.

\section{Acknowledgements}

Not applicable.

\section{Funding}

The present study was supported by grants from Chinese Medicine Pharmaceutical Key Discipline of Shaanxi Province (grant no. 303061107), Natural Science Foundation of China (grant no. 81703720), Key R\&D Program of Shaanxi Province (grant nos. 2019SF-290 and 2018SF-314). Key discipline of traditional Chinese Medicine Pharmaceutical Engineering of Shaanxi Provincial Administration of traditional Chinese Medicine (grant no. 132018004), Discipline Innovation team Project of Shaanxi University of Chinese Medicine (grant no. 2019-YL11), Shaanxi Provincial Department of Education Project (grant no. 18JS026), and the Shaanxi Provincial
Administration of traditional Chinese Medicine Project (grant no. JPC056).

\section{Availability of data and materials}

The datasets used and/or analyzed during the present study are available from the corresponding author upon reasonable request.

\section{Authors' contributions}

YL performed the experiments and data analysis, and wrote the manuscript. JZ performed the experiment and participated in the analysis of the experimental data. YS conceptualized the study and analyzed the experimental data. JT and YW performed experiments. XZ, DG and MY conceptualized the study. All authors read and approved the final manuscript.

\section{Ethics approval and consent to participate}

The study was approved by the Ethical Committee of Shaanxi University of Chinese Medicine.

\section{Patient consent for publication}

Not applicable.

\section{Competing interests}

The authors declare that they have no competing interests.

\section{References}

1. Lam HY, Tergaonkar V and Ahn KS: Mechanisms of allergen-specific immunotherapy for allergic rhinitis and food allergies. Biosci Rep: Apr 30, 2020 (Epub ahead of print). doi: 10.1042/BSR20200256.

2. Mitsias DI, Dimou MV, Lakoumentas J, Alevizopoulos K, Sousa-Pinto B, Fonseca JA, Bousquet J and Papadopoulos NG: Effect of nasal irrigation on allergic rhinitis control in children; complementarity between CARAT and MASK outcomes. Clin Transl Allergy 10: 9, 2020.

3. Rawls M, Thiele J, Adams DE, Steacy LM and Ellis AK: Clinical symptoms and biomarkers of Bermuda grass-induced allergic rhinitis using the nasal allergen challenge model. Ann Allergy Asthma Immunol 124: 608-615.e2, 2020.

4. Wang GA: Efficacy of desloratadine citrate disodium combined with mometasone furoate in the treatment of allergic rhinitis and its effect on patients' complications. Chinese Journal of Rational Drug Use 16: 179-181, 2019 (In Chinese).

5. Yang CS, Xu J and Zhang YY: Determination of 1,8-cineole in bitongning spray by gas chromatography. Guizhou Med J 35: 929-930, 2011 (In Chinese). 
6. Li XB and Wang P: Determination of volatile oil content in bitongning drops by GC method. Heilongjiang Med J 24: 16-17, 2011.

7. Cai XZ: Rudimentary research on identification of Herba Centipedae and screening of its active material anti-Allergic Rhinitis. Southern Medical University, 2008 (In Chinese).

8. Lin YC and Gao M: Research progress on chemical constituents and pharmacology of Centipeda minima. J Zhejiang Chin Med Univ 35: 303-304, 2011

9. Yang XX and Zhuang ZQ: Research progress on chemical constituents and pharmacological action of Flos magnoliae. Chin Tradit Herbal Drugs 7: 490-492, 1998.

10. Yuan JM, Huang XY and Zhou YT: Preparation of Flos magnoliae-Herba Centipedae thermosensitive nasal in situ gels Zhong Cheng Yao 40: 2, 656-662, 2018.

11. Liang YL, Zhang XF and Zou JB: Pharmacology mechanism of and for treating allergic rhinitis based on pharmacology network Drug Dev Ind Pharm 45: 547-555, 2019.

12. Okińczyc P, Szumny A, Szperlik J, Kulma A, Franiczek R, Żbikowska B, Krzyżanowska B and Sroka Z: Profile of polyphenolic and essential oil composition of polish propolis, black poplar and Aspens buds. Molecules 23: E1262, 2018.

13. Katdare A, Khunt D, Thakkar S, Polaka SN and Misra M Comparative evaluation of fish oil and butter oil in modulating delivery of galantamine hydrobromide to brain via intranasal route: Pharmacokinetic and oxidative stress studies. Drug Deliv Transl Res 10: 1136-1146, 2020.

14. Mitsou E, Pletsa V, Sotiroudis GT, Panine P, Zoumpanioti M and Xenakis A: Development of a microemulsion for encapsulation and delivery of gallic acid. The role of chitosan. Colloids Surf B Biointerfaces 190: 110974, 2020.

15. Xu T, Ge SM, Deng LH, Wei MY, Xu YH and Wu CB Preparation of pseudolaric acid B microemulsion and its transdermal penetration. Chin Tradit Herbal Drugs 43: 683-689, 2012

16. Lu SS, Qian YH and Xu BB: Application of microemulsification technology in transdermal penetration of volatile oil from Chinese material medical. Sci Technol Vis 25: 237-239, 2018

17. Wu JY,Li YJ,Han M, Hu XB, Yang L, Wang JM and Xiang DX: A microemulsion of puerarin-phospholipid complex for improving bioavailability: Preparation, in vitro and in vivo evaluations. Drug Dev Ind Pharm 44: 1336-1341, 336-341, 2018.

18. Chen Y, Cheng G, Hu R, Chen S, Lu W, Gao S, Xia H, Wang B, Sun $\mathrm{C}$, Nie X, et al: A nasal temperature and $\mathrm{pH}$ dual-responsive in situ gel delivery system based on microemulsion of huperzine A: Formulation, evaluation, and in vivo pharmacokinetic study. AAPS PharmSciTech 20: 301, 2019.

19. Mishra R, Prabhavalkar KS and Bhatt LK: Preparation, optimization, and evaluation of Zaltoprofen-loaded microemulsion and microemulsion-based gel for transdermal delivery. J Liposome Res 26: 297-306, 2016.

20. Cai H, Ma KY and Li HL: Study on extraction technology of volatile oil from Flos magnoliae. Jiujiang Xueyuan Xuebao Ziran Kexue Ban 32: 8-12, 2017 (In Chinese.

21. Cho YH, Kim S, Bae EK, Mok CK and Park J: Formulation of a cosurfactant-free $\mathrm{O} / \mathrm{W}$ microemulsion using nonionic surfactant mixtures. J Food Sci 73: E115-E121, 2008.

22. Shah A, Thool P, Sorathiya K, Prajapati H, Dalrymple D and Serajuddin AT: Effect of different polysorbates on development of self-microemulsifying drug delivery systems using medium chain lipids. Drug Dev Ind Pharm 44: 215-223, 2018.

23. Song WJ and Gu W: Pharmacology study and prospect of aromatic Chinese herbs. Zhonghua Zhongyiyao Zazhi 32: 2, 609-611, 2017

24. Yang XY and Yi L: Preparation and in vitro transdermal study of zolmitriptan-diclofenac microemulsion. China Pharmacy 28: 1841-1844, 2017.

25. Golmohammadzadeh S, Farhadian N, Biriaee A, Dehghani F and Khameneh B: Preparation, characterization and in vitro evaluation of microemulsion of raloxifene hydrochloride. Drug Dev Ind Pharm 43: 1619-1625, 619-625, 2017.

26. Kotmakçı M, Akbaba H, Erel G, Ertan G and Kantarcı G: Improved method for solid lipid nanoparticle preparation based on hot microemulsions: Preparation, characterization, cytotoxicity, and hemocompatibility evaluation. AAPS PharmSciTech 18 $1355-1365,2017$

27. Akram A, Rasul A, Waqas MK, Irfan M, Khalid SH, Aamir MN, Murtaza G, Ur Rehman K, Iqbal M and Khan BA: Development, characterization and evaluation of in-vitro anti-inflammatory activity of ginger extract based micro emulsion. Pak J Pharm Sci 32: 1327-1332, 2019.
28. Lee J, Lee Y, Kim J, Yoon M and Choi YW: Formulation of microemulsion systems for transdermal delivery of aceclofenac. Arch Pharm Res 28: 1097-1102, 2005

29. Golwala P, Rathod S, Patil R, Joshi A, Ray D, Aswal VK, Bahadur P and Tiwari S: Effect of cosurfactant addition on phase behavior and microstructure of a water dilutable microemulsion. Colloids Surf B Biointerfaces 186: 110736, 2020.

30. Zhang JJ, Cui SF and Tang XL: Study on preparation technology and in vitro transdermal permeability of compound clove microemulsion. Zhong Yao Cai: 608-611, 2007.

31. Liu X, Zhang ZH and Chen Y: Preparation of colchicine microemulsion and its transdermal permeation in vitro. Chin Tradit Herbal Drugs 42: 963-967, 2011.

32. Yang F, Zhou J, Hu X, Yu SK, Liu C, Pan R, Chang Q, Liu X and Liao Y: Preparation and evaluation of self-microemulsions for improved bioavailability of ginsenoside-Rh1 and Rh2. Drug Deliv Transl Res 7: 731-737, 2017.

33. Yang J, Xu H, Wu S, Ju B, Zhu D, Yan Y, Wang M and $\mathrm{Hu}$ J: Preparation and evaluation of microemulsion based transdermal delivery of Cistanche tubulosa phenylethanoid glycosides. Mol Med Rep 15: 109-116, 2017.

34. Zhai XY: Effect of volatile oil from magnolia on Th cells of Guinea pigs with allergic rhinitis. Shaanxi J Tradit Chin Med 31: 116-118, 2010.

35. Liu ZG, Yu HM, Wen SL and Liu YL: Histopathological study on allergic rhinitis treated with Centipeda minima. Zhongguo Zhong Yao Za Zhi 30: 292-294, 2005 (In Chinese).

36. Wu M, Zhang J and Zhang X: Clinical observation of Flos magnoliae volatile oil nano-liposome nasal drops in treating pediatric allergic rhinitis. Zhong guo Zhong Xi Yi Jie He Za Zhi 29: 740-742, 2009.

37. Jia XS and Zhang YY: Research progress on essential oils of Cenpiteda minima (L.) A. Br. Aschers. Shandong Chem Ind 47: 66-68, 2018.

38. Guan Z, Ma XZ and Lv GY: Effects of VOM bp on IL-12, IFN- $\gamma$ and histamine levels of rats with allergic rhinitis. Pharmacol Clin Chin Materia Med 27: 70-72, 2011

39. Wang YQ, Yang YZ, Wu ZF, Xiong YK and Yang M: Traditional function and modern research progress on volatile oil in Chinese materia medica. Chin Tradit Herbal Drugs 49: 455-461, 2018

40. Das S, Lee SH, Chia VD, Chow PS, Macbeath C, Liu Y and Shlieout G: Development of microemulsion based topical ivermectin formulations: Pre-formulation and formulation studies. Colloids Surf B Biointerfaces 189: 110823, 2020.

41. Li SL, Duan Q, Zhao ZD, Xia L, Liu WH, Wang XG and Shen XZ: Preparation and quality evaluation of Acori Tatarinowii Rhizoma-volatile oil microemulsion. Chin Tradit Herbal Drugs 50: 1, 935-941, 2019 (In Chinese).

42. Xu WT: Study on the regularity of the formation of microemulsion by nonionic surfactans. PhD dissertation. Jiangnan University, 2009 (In Chinese).

43. Paulo BB, Alvim ID, Reineccius G and Prata AS: Performance of oil-in-water emulsions stabilized by different types of surface-active components. Colloids Surf B Biointerfaces 190: 110939, 2020.

44. Su J, Wu CH and Jiang F: Preparation of Paeonol self-microemulsion transdermal delivery system with peppermint oil as carrier. Zhongguo Shiyan Fangjixue Zazhi 23: 11-16, 2017 (In Chinese).

45. Yuan ZZ, Yin SY and Jin Y: Preparation and quality evaluation of Eugenia oleifera oil bano-emulsion. Lishizhen Med Materia Med Res 28: 620-622, 2017.

46. Chen JJ, Xia J, Song J, Luo MF and Huang Z: Effect of Eugenol in the microemulsion as an oil excipient and enhancer on percutaneous absorption of Baicalin. Xiandai Shengwu Yixue Jinzhan 16: 410-413, 2016 (In Chinese).

47. Lu SS, Zhao YR, Yao JH, Ren L, Li Y and Chen J: Preparation technology on essential oil from Chinese materia medica as penetration enhancers. Chin Tradit Herbal Drugs 49: 2477-2481, 2018 (In Chinese).

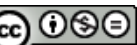

This work is licensed under a Creative Commons Attribution-NonCommercial-NoDerivatives 4.0 International (CC BY-NC-ND 4.0) License. 